\title{
Quasi-Equilibrium in the Mixture of Polystyrene and Poly(methylphenylsiloxane)
}

\author{
Shuichi NoJIMA and Takuhei NOSE \\ Department of Polymer Chemistry, Tokyo Institute of Technology, \\ Ookayama, Meguro-ku, Tokyo 152, Japan.
}

(Received September 16, 1981)

\begin{abstract}
For liquid mixture of polystyrene (PS) and poly(methylphenylsiloxane), we determined cloud points, glass transition temperatures of the homogeneous blends, and the compositions of phases separated at various temperatures in the two-phase region. In this system, phase separation and glass transition occur in ranges of comparable temperature. For this characteristic, one of the separated phases, which was rich in PS, did not reach equilibrium compositions but approached quasi-equilibrium compositions. A theory has been developed to examine the effect of the settling-down of one phase to a quasi-equilibrium composition on the composition of the conjugate phase.

KEY WORDS Polymer Blend / Quasi-Equilibrium /Phase Separation / Cloud Point / Glass Transition /
\end{abstract}

Usually, a binary polymer mixture in the partially compatible region exhibits two glass transition temperatures $T_{\mathrm{g}}$ corresponding to the compositions of the separated phases. Thus, $T_{\mathrm{g}}$ measurement can be utilized to judge the compatibility of polymer blends and to estimate the composition of partially compatible polymer blends. ${ }^{1-4}$ In applying this method to the determination of the equilibrium composition of separated phases, however, it is necessary that we are concerned with the phase separation at the temperature far above the $T_{\mathrm{g}} v s$. composition curve for the system studied. Otherwise, observation cannot be made of equilibrium phase separation within experimentally accessible time due to the low mobility of polymer molecules in the glassy state.

This paper deals with a mixture of two liquid polymers in which the cloud point curve and the $T_{\mathrm{g}}$ $v s$. composition curve appear in ranges of comparable temperature. If this polymer blend is quenched from a temperature above the cloud point curve to a temperature $T_{0}$ in the two-phase region at a very fast rate, phase separation actually starts taking place when $T_{0}$ has been reached, but one of the separated phases, which is rich in the component with higher $T_{\mathrm{g}}$, cannot approach an equilibrium composition within an accessible period of time.
Instead, it eventually takes on and stays at a certain composition different from the equilibrium composition. By thermodynamic analysis it is found that this settling-down of one phase to a quasiequilibrium composition effects the composition of the conjugate phase rich in the component with lower $T_{\mathrm{g}}$. On the other hand, if the blend is cooled very slowly toward $T_{0}$, there appears at each temperature down to $T_{0}$ a new phase again rich in the component with higher $T_{\mathrm{g}}$ and a settling-down to a quasi-equilibrium composition. Thus, on reaching $T_{0}$, the blend is a mixture consisting of a series of phases; one phase rich in lower- $T_{\mathrm{g}}$ component and the others (rich in higher- $T_{\mathrm{g}}$ component) with continuously varying compositions which may approximately follow the $T_{\mathrm{g}} v s$. composition curve.

In the present paper, the former case is studied, i.e., the rapid quenching of a blend to the two-phase region. Liquid mixtures of low-molecular-weight polystyrene (PS) and poly(methylphenylsiloxane) (PMPS) were chosen for the experiment. Some mixtures have been reported in which the phase separation and glass transition occur in ranges of comparable temperature. ${ }^{4-8}$ In these mixtures, however, crystallization occurs during the liquidliquid phase separation, giving rise to various 
complications which the present system avoids, since both polymers are non-crystalline. The compositions of the separated phases were determined as functions of initial composition, separation temperature, and separation time, together with the cloud point curve and $T_{\mathrm{g}} v s$. composition relationship. Furthermore, a thermodynamic theory has been developed on the effect exerted on the composition of one phase when that of the conjugate phase is frozen at a value different from the equilibrium composition.

\section{EXPERIMENTAL}

\section{Materials}

The polystyrene (PS) sample used was a product of Pressure Chemical Co., and the weight-average molecular weight $M_{w}$ and the ratio of $M_{w}$ to the number-average molecular weight $M_{n}$ were 9,000 and about 1.06 , respectively. The poly(methylphenylsiloxane) (PMPS) used was a commercial product of Toray Silicone, and dried for $50 \mathrm{~h}$ under vacuum to eliminate impurities before use. Its $M_{w}$ and $M_{w} / M_{n}$ determined by gel permeation chromatography were 2,800 and 1.57 , respectively.

\section{Preparation of Blends}

Two methods were used to prepare mixtures of these two polymers, depending on the composition, which is here expressed in terms of the weight percent of PS. For higher compositions, both polymers were dissolved in a common solvent benzene at a total concentration less than $25 \mathrm{wt} \%$. The solution was then cast on a glass plate, and the solvent was evaporated by heating for $50 \mathrm{~h}$ at $110^{\circ} \mathrm{C} .{ }^{9}$ For lower compositions, the two polymers were directly mixed above $120^{\circ} \mathrm{C}$. The blends prepared by these two methods gave a consistent cloud point curve.

\section{Determination of Cloud Points and $T_{\mathrm{g}}$ vs. Composition Relation}

Cloud points were determined by measuring the change in intensity of light transmitted through a sample cell with an optical path of about $1 \mathrm{~mm}$. The temperature $T_{\mathrm{c}}(R)$ at which the sample started becoming turbid when cooled at a rate $R$ was measured for various $R$ from 0.02 to $0.3^{\circ} \mathrm{C} \mathrm{min}{ }^{-1}$, and the data were extrapolated to $R=0$ to obtain the cloud point $T_{\mathrm{c}}(0)$. Figure 1 illustrates the $T_{\mathrm{c}}(R)$

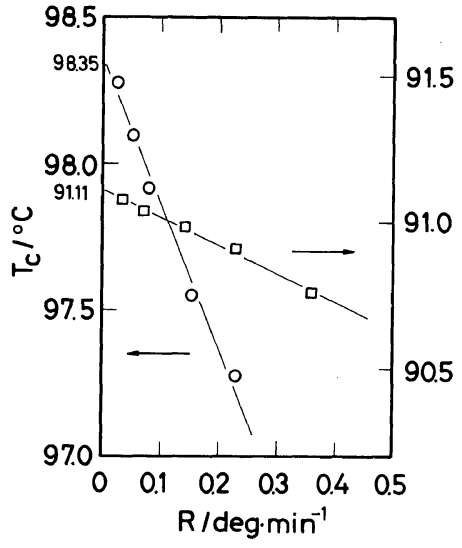

Figure 1. Cloud point temperature $T_{\mathrm{c}}$ as a function of cooling rate $R$ : $\bigcirc, 47.4 \mathrm{wt} \% \mathrm{PS} ; \square, 32.5 \mathrm{wt} \%$ PS.

data for the two blends. The slope of $T_{\mathrm{c}}(R)$ against $R$, which was linear for all cases examined, varied with the composition of the blend. In the PMPSrich region, it first decreased, reached a minimum at $30 \mathrm{wt} \%$, and then increased as the PS content was increased. Turbid blends became immediately transparent when warmed above $T_{\mathrm{c}}(0)$.

To determine the $T_{\mathrm{g}}$ of a homogeneous blend of known composition the sample maintained above $110^{\circ} \mathrm{C}$ was quenched to the temperature of liquid nitrogen, $-196^{\circ} \mathrm{C}$, and subjected to a differential scanning calorimetry (DSC) measurement using a Parkin-Elmer DSC Model II and a heating rate of

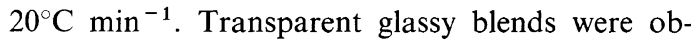
tained only in the PS-rich region. As illustrated in Figure 3, the DSC thermograms obtained exhibited two breaks which may be defined as the points of intersection of the upper and lower base lines with the tangent at the inflection point. The glass transition temperatures corresponding to these breaks are denoted by $T_{\mathrm{g}}{ }^{+}$and $T_{\mathrm{g}}{ }^{-}$, as indicated in Figure 3.

\section{Composition Determination of Separated Phases}

A blend of known composition was cooled from $120^{\circ} \mathrm{C}$ to a desired temperature $T_{0}$ below the cloud point curve at a rate as fast as $320^{\circ} \mathrm{C} \mathrm{min}^{-1}$, maintained at $T_{0}$ for a prescribed period of time and then quenched to $-73^{\circ} \mathrm{C}$ in the DSC apparatus for a DSC measurement. This experiment was carried out for two blends of 50.4 and $55.0 \mathrm{wt} \%$ at various $T_{0}$ down to values near $T_{\mathrm{g}}$ for these compositions, with $20 \mathrm{~h}$ for $55.0 \mathrm{wt} \%$ and $30 \mathrm{~min}$ and $20 \mathrm{~h}$ for 50.4 
$\mathrm{wt} \%$ as the period of time for the phase separation. Actually, the phase separation for $30 \mathrm{~min}$ was made in the DSC apparatus, and that for $20 \mathrm{~h}$ in an external thermostat. In all cases, the DSC measurements on the phase-separated blends were carried out at a heating rate of $20^{\circ} \mathrm{C} \mathrm{min}{ }^{-1}$. The compositions in the separated phases were determined by $T_{\mathrm{g}}{ }^{+}$and $T_{\mathrm{g}}{ }^{-}$on the DSC thermogram so obtained.

\section{RESULTS AND DISCUSSION}

Cloud Point Curve and $T_{\mathrm{g}}$ vs. Composition Relation Figure 2 shows the cloud point curve obtained.

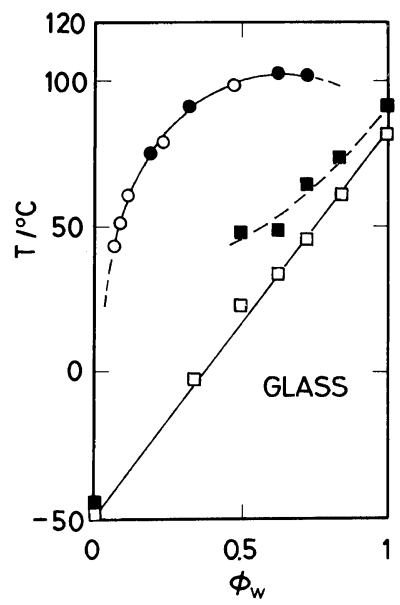

Figure 2. Cloud points and glass transition temperatures: blend cast from benzene solution; $\bigcirc$, blend mixed directly; $\square$, glass transition temperature obtained from $T_{\mathrm{g}}{ }^{-} ; \boldsymbol{\square}$, glass transition temperature obtained from $T_{\mathrm{g}}{ }^{+}$
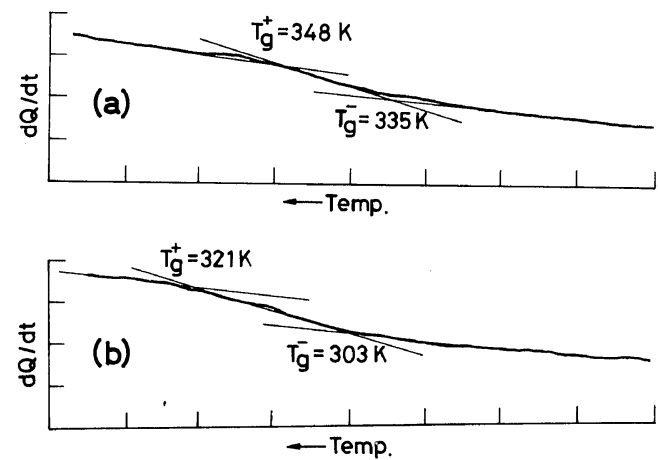

Figure 3. DSC thermograms of the homogeneous blends: a), $84.0 \mathrm{wt}^{\circ} \%$ PS; b), $62.4 \mathrm{wt} \%$ PS.
When the PS composition $\phi_{\mathrm{w}}$ was above $75 \mathrm{wt} \%$, no inception of turbidity was observed even at the slowest cooling rate of $0.02^{\circ} \mathrm{C} \mathrm{min}^{-1}$.

Figure 2 also displays $T_{\mathrm{g}}{ }^{+}$and $T_{\mathrm{g}}{ }^{-}$of homogeneous blends as functions of $\phi_{\mathrm{w}}$. It is evident that $T_{\mathrm{g}}{ }^{-}$varies linearly with $\phi_{\mathrm{w}}$ throughout the entire composition range, yielding $-49( \pm 1)^{\circ} \mathrm{C}$ for pure PMPS and $82( \pm 2)^{\circ} \mathrm{C}$ for pure PS; the latter is a little lower than the literature values. ${ }^{10,11} \mathrm{As} \phi_{\mathrm{w}}$ is lowered, the spread of $T_{\mathrm{g}}$, i.e., $T_{\mathrm{g}}{ }^{+}-T_{\mathrm{g}}{ }^{-}$increases, as can be seen from Figure 3 which also shows the DSC thermograms for different $\phi_{\mathrm{w}}, 84.0 \mathrm{wt} \%$ (a) and $62.4 \mathrm{wt}^{\circ} \%$ (b). At $\phi_{\mathrm{w}}=35 \mathrm{wt} \%$, the DSC thermogram exhibited, in addition to $T_{\mathrm{g}}{ }^{+}$and $T_{\mathrm{g}}{ }^{-}$, another glass transition which could be assigned to pure PMPS.

In Figure 2, attention should be directed to the fact that the phase separation and glass transition of the present system of liquid polymers occur in ranges of comparable temperature.

The appearence of two glass transition temperatures $T_{\mathrm{g}}{ }^{+}$and $T_{\mathrm{g}}{ }^{-}$may be explained as follows. While being quenched to liquid nitrogen temperature for DSC measurement, a blend should undergo partial phase separation. As the temperature is lowered, the PMPS-rich phase evolved becomes richer in PMPS, smaller in size, and eventually no longer detectable by DSC. ${ }^{12,13}$ However, a series of PS-rich phases that appear at successive temperatures in this process are frozen by vitrification. This indicates that the quenched blend contains a range of PS-rich phases that varying in compositions and $T_{\mathrm{g}}{ }^{+}$and $T_{\mathrm{g}}{ }^{-}$correspond to the upper and lower bounds of this range, respectively. ${ }^{14,15}$ It is noted that the lower bound corresponding to $T_{\mathrm{g}}{ }^{-}$is the composition of the initial homogeneous blend, since the PS composition of PS-rich phases separated during the quenching is always higher than that of the initial blend.

Two reasons may be given for the composition change in $T_{\mathrm{g}}{ }^{+}-T_{\mathrm{g}}{ }^{-}$as shown in Figure 2. First, as the PS composition is lowered, the blend passes through a longer two-phase liquid region until quenched to the liquid nitrogen temperature. This facilitates the separation of phases richer in PS in the blend, thus leading to a greater $T_{\mathrm{g}}{ }^{+}-T_{\mathrm{g}}{ }^{-}$. Second, the average rate of the phase separation should be larger for lower PS compositions, since the phases undergo separation more easily at such compositions in a less glassy state with high mo- 

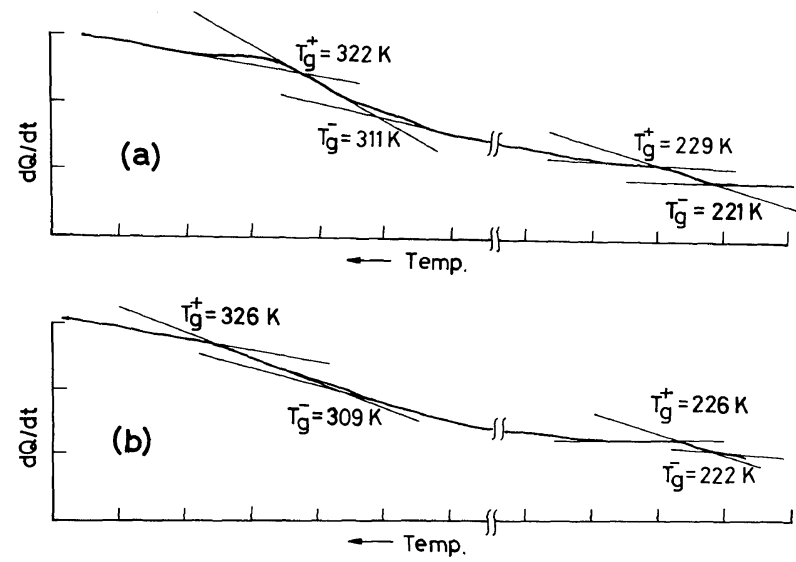

Figure 4. DSC thermograms of the phase-separated blends. Initial composition is $50.4 \mathrm{wt}_{\mathrm{o}}^{\mathrm{o}} \mathrm{PS}$ : a), annealed at $37^{\circ} \mathrm{C}$ for $30 \mathrm{~min}$; b), annealed at $77^{\circ} \mathrm{C}$ for $30 \mathrm{~min}$.

bility of the polymer molecules.

\section{Compositions of Separated Phases}

Figure 4 illustrates the DSC thermograms for $50.4 \mathrm{wt}^{\%} \%$ blend subjected to phase separation at $37^{\circ} \mathrm{C} \mathrm{(a)} \mathrm{and} 77^{\circ} \mathrm{C}$ (b) for a period of $30 \mathrm{~min}$. For each of these thermograms, the $T_{\mathrm{g}}{ }^{+}$and $T_{\mathrm{g}}{ }^{-}$on the left side were assignable to PS-rich phases of varying compositions; those on the right side were assigned to pure PMPS. No $T_{\mathrm{g}}{ }^{+}$or $T_{\mathrm{g}}{ }^{-}$assignable to the PMPS-rich phases could be seen. This suggests that the PMPS-rich phase was separated further into pure components when the blend was quenched for DSC measurement.

In Figure 5 are shown the compositions calculated from $T_{\mathrm{g}}{ }^{+}$and $T_{\mathrm{g}}{ }^{-}$for the PS-rich phases of the blend of $50.4 \mathrm{wt} \%$ subjected to phase separation at various $T_{0}$ for $30 \mathrm{~min}$. The horizontal bar in the figure indicates the composition spread obtained when the same blend was quenched directly at the temperature of liquid nitrogen. In deriving these results, it was assumed that $T_{\mathrm{g}}{ }^{-}$varies with composition, following the solid straight line in Figure 2 , and that $\mathrm{T}_{\mathrm{g}}^{+}$also varies linearly with composition, following the straight line connecting the filled square points for pure PS and PMPS in Figure 2 and indicated by a broken line in Figure 5.

It can be seen that the compositions from $T_{\mathrm{g}}{ }^{+}$at different $T_{0}$ are almost constant and equal to the upper limit of the bar. This implies that the phases corresponding to $T_{\mathrm{g}}{ }^{+}$evolved not during the standing at $T_{0}$, but during the subsequent quenching for

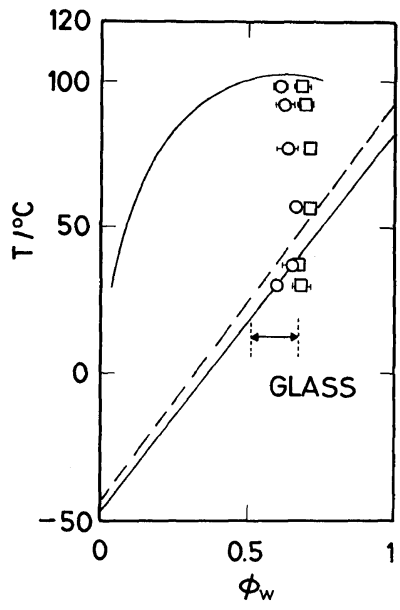

Figure 5. The compositions of phases separated for $30 \mathrm{~min}$. Initial composition is $50.4 \mathrm{wt} \% \mathrm{PS}: \mathrm{O}$, obtained from $T_{\mathrm{g}}{ }^{-} ; \square$, obtained from $T_{\mathrm{g}}{ }^{+}$. The bar $(\leftrightarrow)$ indicates composition distribution when the homogeneous blend is quenched into the glassy state.

DSC measurements. The compositions from $T_{\mathrm{g}}{ }^{-}$ also are almost independent of $T_{0}$, but are higher than the lower limit of the bar corresponding to the composition of the given homogeneous blend, 50.4 $\mathrm{wt} \%$ in the present case. Thus, the compositions from $T_{\mathrm{g}}{ }^{-}$should represent those of the PS-rich phases separated during the standing at $T_{0}$ for $30 \mathrm{~min}$. The fact that they are independent of $T_{0}$ may be explained as follows.

Roughly speaking, the rate of phase separation is 
determined by the product of the mobility of component polymers in the blend and the instability of the system. ${ }^{16}$ The latter is expressed in terms of the second derivative of the free energy with respect to the composition in the early stage of spinodal decomposition and is enhanced by lowering the temperature at which phase separation is allowed to take place. Thus, thermodynamic instability accelerates phase separation when carried out at lower temperature. However, in the present system, the mobility of polymer molecules is more suppressed at lower temperature, since the system approaches the glassy state more closely. This effect retards the evolution of phase separation. These two oppositely acting effects determine the ratio of phase separation, and hence the composition from $T_{\mathrm{g}}{ }^{-}$, approximately independent of $T_{0}$.

Figure 6 shows the compositions of the PS-rich phases calculated from $T_{\mathrm{g}}{ }^{+}$and $T_{\mathrm{g}}{ }^{-}$for the blends of $50.4 \mathrm{wt} \%$ and $55.0 \mathrm{wt} \%$ subjected to phase separation at various $T_{0}$ for $20 \mathrm{~h}$. The data points appear to the right of the corresponding dotted curves which fit the data points shown in Figure 5. This fact indicates that further phase separation occurred when the blend was left at $T_{0}$ for a longer period of time, as can be expected. The compositions from $T_{\mathrm{g}}{ }^{-}$at higher $T_{0}$ shift to the right more from the corresponding dotted curve. This phenomenon is consistent with fact that the mo-

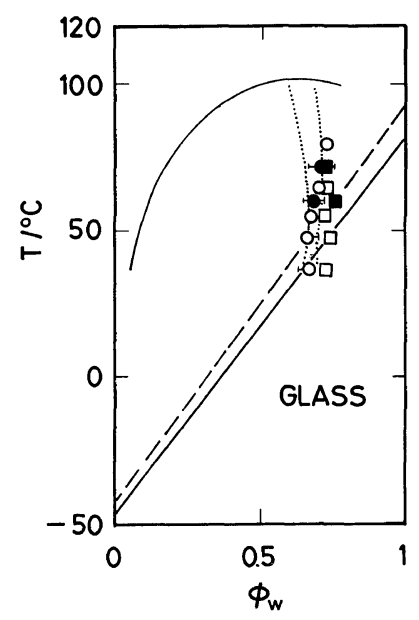

Figure 6. The composition of phases separated for $20 \mathrm{~h}$ : initial composition of PS, $50.4 \mathrm{wt} \%(O, \square)$ and $55.0 \mathrm{wt} \%(\boldsymbol{\square}) ; \bigcirc, \boldsymbol{\square}$; obtained from $T_{\mathrm{g}}{ }^{-}, \square, \mathbf{\square}$; obtained from $T_{\mathrm{g}}{ }^{+}$. bility of polymer molecules is greater for higher $T_{0}$.

In Figure 6, we see that the compositions, calculated from either $T_{\mathrm{g}}{ }^{+}$or $T_{\mathrm{g}}{ }^{-}$, for blends of 50.4 and $55.0 \mathrm{wt} \%$ fall approximately on a single curve. We carried out similar phase separation experiments at various $T_{0}$ with a standing time of $20 \mathrm{~h}$ for two blends of 25 and $35 \mathrm{wt} \%$, and found that the compositions fell on the same curve. These results indicate that the compositions of the PS-rich phase separated at a given temperature for a period of $20 \mathrm{~h}$ are essentially independent of the composition of the initial homogeneous blend. These differ considerably from the equilibrium compositions predictable from the cloud point curve, and simply represent quasi-equilibrium compositions. The attainment of true equilibrium compositions for the values of $T_{0}$ chosen in this work would far exceed the time allowable for our experiments.

\section{Theory}

Suppose that a binary polymer blend is rapidly cooled to a temperature in the two-phase region and that one of the separated phases is frozen at a certain composition different from the equilibrium composition corresponding to the fixed temperature. It may be asked what deviation from the equilibrium composition of the conjugate phase occurs when the mobility of the polymer chains in this phase is sufficiently high.

The frozen-in phase and its conjugate phase are denoted by double and single primes, respectively. The equilibrium between these two phases at constant temperature and pressure is established when the chemical potentials $\mu_{1}$. and $\mu_{2}$ satisfy the relation,

$$
\begin{aligned}
\mathrm{d} G & =\left(\mu_{1}{ }^{\prime} \mathrm{d} n_{1}+\mu_{2}{ }^{\prime} \mathrm{d} n_{2}\right)-\left(\mu_{1}{ }^{\prime} \mathrm{d} n_{1}+\mu_{2}{ }^{\prime \prime} \mathrm{d} n_{2}\right) \\
& =\left(\mu_{1}{ }^{\prime}-\mu_{1}{ }^{\prime}\right) \mathrm{d} n_{1}+\left(\mu_{2}{ }^{\prime}-\mu_{2}{ }^{\prime}\right) \mathrm{d} n_{2}=0
\end{aligned}
$$

where the subscripts 1 and 2 denote the components of the blend, and $\mathrm{d} n_{i}$ is an infinitesimal amount of component $i$ actually transferred from the double prime to the single prime phase. Since, in the present case, the composition of the double prime phase is fixed at a certain value, say $\phi_{\mathrm{g}}, \mathrm{d} n_{1}$ and $\mathrm{d} n_{2}$ are not allowed to undergo independent change and must obey the restriction,

$$
x_{1} \mathrm{~d} n_{1} /\left(x_{1} \mathrm{~d} n_{1}+x_{2} \mathrm{~d} n_{2}\right)=\phi_{\mathrm{g}}
$$

where $x_{i}$ is the number of segments in component $i$. 


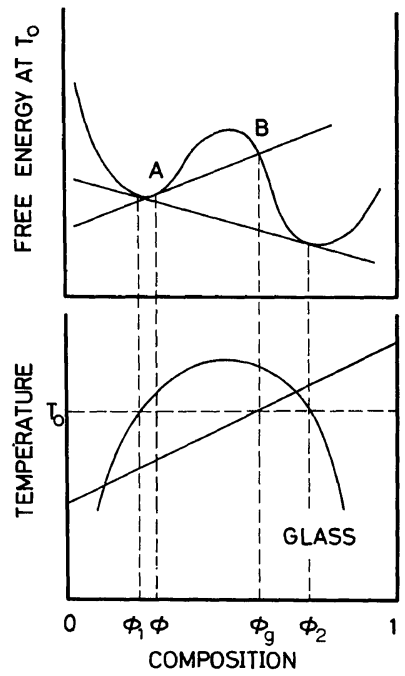

Figure 7. Deviation of a quasi-equilibrium composition from the equilibrium composition with the free energy vs. composition curve.

From eq 1 and 2 we obtain as the equilibrium condition

$$
\left(\mu_{1}{ }^{\prime}-\mu_{1}{ }^{\prime \prime}\right) \phi_{\mathrm{g}} / x_{1}+\left(\mu_{2}{ }^{\prime}-\mu_{2}{ }^{\prime \prime}\right)\left(1-\phi_{\mathrm{g}}\right) / x_{2}=0
$$

This permits computation of the composition $\phi$ of the single prime phase as a function of temperature for a given temperature dependence of $\phi_{\mathrm{g}}$.

In the free energy $v s$. composition curve delineated in Figure 7, point B corresponds to $\phi_{\mathrm{g}}$ and the line passing through this point touches the free energy curve at point $\mathrm{A}$. According to eq 3 , the composition at point $\mathrm{A}$ is equal to the quasiequilibrium composition $\phi$ of the single prime phase which deviates from the equilibrium one $\phi_{1}$.

We substitute Scott's expression for the free energy of mixing $\Delta G,{ }^{17}$ i.e.,

$$
\begin{aligned}
\Delta G= & R T\left[\left(\phi / x_{1}\right) \ln \phi+\left\{(1-\phi) / x_{2}\right\} \ln (1-\phi)\right. \\
& +\chi \phi(1-\phi)]
\end{aligned}
$$

into eq 3 . Here $\chi$ denotes the Flory-Huggins interaction parameter. The result reads

$$
\begin{aligned}
& {\left[\left(1-\phi_{\mathrm{g}}\right) / x_{2}\right] \ln \left[(1-\phi) /\left(1-\phi_{\mathrm{g}}\right)\right]} \\
& +\left(\phi_{\mathrm{g}} / x_{1}\right) \ln \left(\phi / \phi_{\mathrm{g}}\right)+\left(\phi_{\mathrm{g}}-\phi\right)\left(1 / x_{1}-1 / x_{2}\right) \\
& +\chi\left(\phi_{\mathrm{g}}-\phi\right)^{2}=0
\end{aligned}
$$

To calculate $\phi$ as a function of $\chi$ from eq 5, we

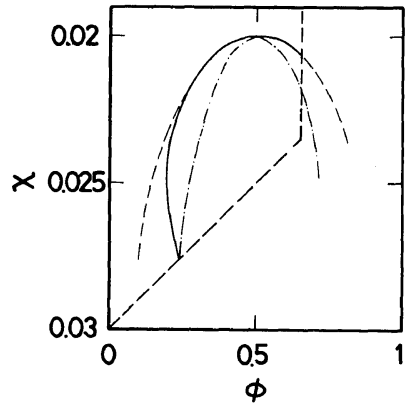

Figure 8. Quasi-equilibrium binodal calculated from eq 5 for variables indicated in the text: - - , quasiequilibrium binodal curve; ---, equilibrium binodal curve; - - - - - spinodal curve.

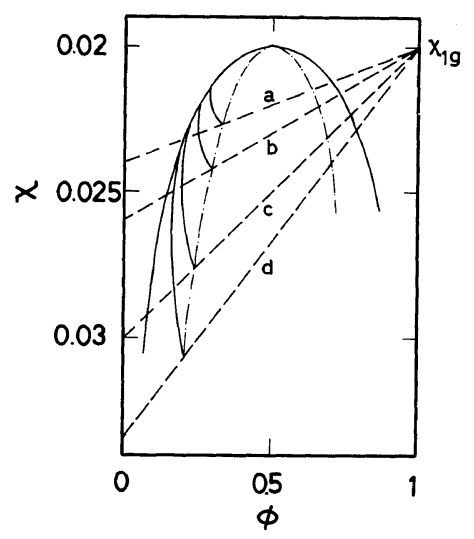

Figure 9. $\chi_{2 \mathrm{~g}}$ dependence of the binodal: $x_{1}=x_{2}=100$, $\chi_{1 \mathrm{~g}}=0.02$, and $\chi_{2 \mathrm{~g}}=$ (a) 0.024 , (b) 0.026 , (c) 0.030 , (d) 0.034 .

take $x_{1}=100, x_{2}=100, \chi_{1 \mathrm{~g}}=0.02$, and $\chi_{2 \mathrm{~g}}=0.03$, where $\chi_{1 \mathrm{~g}}$ and $\chi_{2 \mathrm{~g}}$ are the values of $\chi$ at the glass transition temperatures for components 1 and 2 , respectively, and assume by referring to the experimental results shown in Figure 6 that $\phi_{\mathrm{g}}$ varies with $\chi$ as indicated by the thick broken line in Figure 8 . The calculated quasi-equilibrium binodal is shown by the solid curve in Figure 8. This curve lies inside the equilibrium binodal represented by a thin broken curve and intersects the spinodal represented by a dot-dash curve at a point on the $\chi$ vs. $\phi_{\mathrm{g}}$ line.

Figure 9 illustrates the change in the quasiequilibrium binodal as the $T_{\mathrm{g}}$ 's of the pure components approach each other. In calculating these curves, it was assumed that $T_{\mathrm{g}}$ varies with $\phi$ according to the Fox relation ${ }^{18}$ for glass transition tem- 
perature of binary mixture. It can be seen that at a given temperature the miscibility region on the left side of the quasi-equilibrium binodal spreads as $\chi_{2 \mathrm{~g}}$ approaches $\chi_{1 \mathrm{~g}}$.

It should be noted that the present model may be too much of a simplification to warrant application to real systems in estimating quasi-equilibrium composition, since the assumption of the high mobility of polymer chains for the single prime phase may, for example, break down near the glass transition region in real systems.

\section{REFERENCES}

1. D. R. Paul and S. Newman, Ed., "Polymer Blends," Vol. 1, Academic Press, New York, N.Y., 1978.

2. O. Olabishi, L. M. Robeson, and M. T. Shaw, "Polymer-Polymer Miscibility," Academic Press, New York, N.Y., 1979.

3. F. P. Price, J. Polym. Sci., Polym. Symp., No. 63, 13 (1978).

4. T. K. Kwei, G. D. Patterson, and T. T. Wang, Macromolecules, 9, 780 (1976).

5. M. Bank, J. Leffingwell, and C. Thies, J. Polym. Sci.,
A-2, 10, 1097 (1972).

6. P. T. van Emmerik and C. A. Smolders, J. Polym. Sci., C, 38, 73 (1972).

7. P. T. van Emmerik and C. A. Smolders, Eur. Polym. J., 9, 293 (1973).

8. E. Roerdink and G. Challa, Polymer, 21, 1161 (1980).

9. M. Bank, J. Leffingwell, and C. Thies, Macromolecules, 4, 43 (1971).

10. S. Krause and M. Iskandar, "Multiphase Polymers," S. L. Cooper and G. M. Esters, Ed., Adv. Chem. Ser., 176, 1979.

11. J. M. G. Cowie, Eur. Polym. J., 11, 297 (1975).

12. W. J. MacKnight, F. E. Karasz, and J. R. Fried Chapter 5 in ref 1.

13. J. Bares, Macromolecules, 8, 244 (1975).

14. J. R. Fried, F. E. Karasz, and W. J. MacKnight, Macromolecules, 11, 150 (1978).

15. D. M. Leitz, L. W. Kleiner, and P. G. Gertenbach, Thermochim. Acta, 35, 51 (1980).

16. See, for example, I. Prigogine and R. Defay, "Chemical Thermodynamics," Longmans, London, 1954, Chapters 16 and 17.

17. R. L. Scott, J. Chem. Phys., 17, 279 (1949).

18. T. G. Fox, Bull. Am. Phys. Soc., 1, 123 (1956). 